\title{
Reply to "Can Patients with Multiple Breast Cancers in the Same Breast Avoid Mastectomy by Having Multiple Lumpectomies to Achieve Equivalent Rates of Local Breast Cancer Recurrence? Response to the Preliminary Alliance 11102 Trial Report"
}

\author{
Kari M. Rosenkranz, MD ${ }^{1}$, Karla Ballman, $\mathrm{PhD}^{2}$, Linda McCall, $\mathrm{MS}^{3}$, Charlotte Dai Kubicky, $\mathrm{MD}^{4}$, \\ Laurie Cuttino, $\mathrm{MD}^{5}$, Huong Le-Petross, $\mathrm{MD}^{6}$, Kelly Hunt, $\mathrm{MD}^{7}$, Armando Giuliano, $\mathrm{MD}^{8}$, \\ Kimberly Van Zee, $\mathrm{MD}^{9}$, Bruce Haffty, $\mathrm{MD}^{9}$, and Judy Boughey, $\mathrm{MD}^{10}$
}

${ }^{1}$ Dartmouth Hitchcock Medical Center, Lebanon; ${ }^{2}$ Division of Biostatistics and Epidemiology, Weill Cornell Medical College, New York City; ${ }^{3}$ Alliance Statististics and Data Center, Duke Cancer Institute, Durham; ${ }^{4}$ Oregon Health and Science University, Portland; ${ }^{5}$ Virginia Commonwealth Universtiy, Richmond; ${ }^{6}$ Diagnostic Radiology, UT MD Anderson Cancer Center, Houston; ${ }^{7}$ Department of Surgical Oncology, UT MD Anderson Cancer Center, Houston; ${ }^{8}$ Surgical Oncology, Cedars-Sinai Medical Center, Los Angeles; ${ }^{9}$ Radiation Oncology, Cancer Institute of New Jersey/Robert Wood, Johnson University Hospital, New Brunswick; ${ }^{10}$ Mayo Clinic, Rochester

\section{To the Editors}

We thank Dr. Winters and Benson for their letter to the editor entitled "Can patients with multiple breast cancers in the same breast avoid mastectomy by having multiple lumpectomies to achieve equivalent rates of local regional recurrence? Response to the preliminary Alliance 11102 trial report". The authors raise some important issues regarding long-term cosmetic outcome (particularly given the low utilization of oncoplastic techniques in our patient cohort) and the potential for long-term radiation complications, given the need for boost to two or three lumpectomy cavities. We agree wholeheartedly that these are potential issues for women undergoing breast-conserving therapy for multiple ipsilateral breast cancer (MIBC). Both of these planned secondary endpoints will be reported in the future. Additionally, we are evaluating the incidence of lymphedema and the primary endpoint of local recurrence in our patient population, which will also be reported in the future.

We acknowledge that the single-arm design may be a limitation in the final analysis of local recurrence rates. A randomized study would provide higher-level evidence and allow comparison of outcomes of breast conservation versus mastectomy in this population. However, it was felt that accrual to a randomized study would be challenging due to potential patient and/or surgeon preference. We congratulate Drs. Winters and Benson and colleagues on their upcoming randomized, prospective MIAMI trial, which evaluates the questions of feasibility and safety of breast conservation in MIBC in a cohort of 50 patients.

(C) Society of Surgical Oncology 2018

First Received: 22 October 2018;

Published Online: 12 December 2018

K. M. Rosenkranz, MD

e-mail: kari.m.rosenkranz@hitchcock.org 